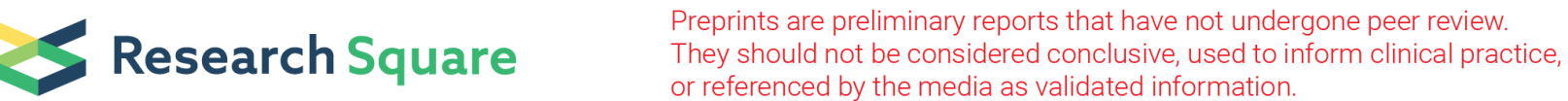

\section{The Baseline Serum Uric Acid as an Indicator of Chemotherapy-Related Adverse Reaction and Mortality in Aged Males with Stage IIIB or IV Non- Small Cell Lung Cancer}

\section{Xiaoyan Chen}

Zigong Mental Health Center

\section{Lisha Hou}

National Clinical Research Center for Geriatrics, Sichuan University

Jianqun Li

Hospital囚T.C.M囚affiliated to Southwest medical university

\section{Yanjiao Shen}

MAGIC China Center, Chinese Evidence-Based Medicine Center, Sichuan University

\section{Fucha Tan}

Affiliated Hospital of Heze Medical College

\section{Birong Dong}

National Clinical Research Center for Geriatrics, Sichuan University

Qiukui Hao ( $\nabla$ haoqiukui@gmail.com )

National Clinical Research Center for Geriatrics $₫$ West China Hospital, Sichuan University

\section{Research Article}

Keywords: serum uric acid, non-small cell lung cancer, chemotherapy, mortality,

Posted Date: September 22nd, 2021

DOI: https://doi.org/10.21203/rs.3.rs-731335/v2

License: (c) (i) This work is licensed under a Creative Commons Attribution 4.0 International License. Read Full License 


\section{Abstract}

Objective: To evaluate the accuracy of baselineserum uric acid(BSUA) in estimating adverse effects (AE) and all-cause mortality (ACM) in older males with stage IIIB or IV non-small cell lung cancer (NSCLC) diagnosis.

Study design:This is a single-center retrospective examination, conducted at the West China Hospital, Sichuan University in Chengdu, Sichuan Province, China, between the duration of January 2010 and December 2017.

Primary outcome and measures:: All patients data was obtained based on medical reports and mortality information was gathered via telephone interviews. BUSA was assessed prior to chemotherapy. Additionally, the end points of this study included chemotherapy-mediated AE and ACM. Binarylogistic regression analysis was used to explore the correlation between BSUA and AE. Lastly, Cox regression analysis was utilized to examine theimpactof BSUA on ACM.

Results: 317 male patients with NSCLC were eligible for this study. Within this population, $18.3 \%$ had stage IIIB and $81.7 \%$ had stage IV NSCLC. Moreover, $81.39 \%$ suffered from adenocarcinoma lung cancer (ACLC), whereas $18.61 \%$ suffered from squamous cell carcinoma lung cancer (SCCLC). As of March 1 , 2019, 257 (81.07\%) patients expired. Following the initial chemotherapeutic course, short-term AE like bone marrow suppression, all infection, liver dysfunction, and digestive reactions, wereobserved in $13.25 \%, 7.26 \%, 5.36 \%$, and $4.1 \%$ of cases, respectively. Upon normalizing with confounding factors, the adjustedlogistic regression model demonstrated thatthe moderate BSUA was independently linked to a lower risk of bone marrow suppression ( $\mathrm{OR}=0.407,95 \% \mathrm{Cl}: 0.178-0.931 ; \mathrm{p}=0.033)$. Moreover, based on the Cox regression analysis, moderate BSUAwas also independently correlated with a low mortality risk $(\mathrm{HR}=0.705,95 \% \mathrm{Cl}: 0.518-0.959 ; \mathrm{p}=0.026)$.

Conclusionखln males patients withstage IIIB or IV NSCLC, BSUA is intimately linked to chemotherapydriven AE and ACM.

\section{Introduction}

Lung cancer (LC) is increasingly common in tumor patients. Moreover, LC mortality is ranked first in China, accounting for 45.9 deaths per 100,000 individuals, among which male deaths $(61.5$ per 100,000) are higher than female deaths $(29.4$ per 100,000$)$ (1). According to Globocan 2020 , China accounts for $39.8 \%$ of LC deaths (2). Non-small cell lung cancer (NSCLC), on the other hand, contributes to $85 \%$ of LC deaths (3-4). This is likely because NSCLC diagnosis often occurs at an advanced clinical stage, and only $53 \%$ of the advanced-stage (i.e. stage III and IV) NSCLC patients undergo chemotherapy (5). Till date, chemotherapy has shown great potential in enhancing NSCLC patient prognosis, with a $<20 \% 5$-year patient survival rate (6-8). However, for purposes of enhancing survival of NSCLC patients, continued exploration into the mechanisms involved are necessary. 
Based on prior reports, the established indicators of NSCLC prognosis include patient characteristics (smoking habit, comorbidities, and thinness) and tumour characteristics (histological grade and clinical stage) (9). In recent years, it was reported that BSUA is related to LC prognosis. One study revealed that elevated baseline serum uric acid (BSUA) worsened survival rates of NSCLC patients (9). Interestingly, another report correlated low BSUA with enhanced mortality from LC. However, this correlation was not established in other forms of cancer (10). In yet another research study, BSUA was shown to have no involvement with patient survival at all, after chemotherapy(11). To further complicate matters, the uric acid mechanism of action is also under dispute. Uric acid is the end-product of nucleotide metabolism and serves a crucial role in antioxidant activities (12). Masi et al. reported that BSUA is a potent antioxidant that eliminates free radicals. Hence, a rise in uric acid can potentially inhibit lipid peroxidation and exert an anti-tumor effect in the human body (12). However, some scholars believe that uric acid has pro-inflammatory properties. Inflammatory mediators and cell effectors are essential components in tumor environments, and, therefore, an inflammatory response, mediated by BSUA, can further promote the proliferation and survival of tumor cells $(13-14)$.

These data contradictions demand additional examination of the link between BSUA and NSCLC patient survival. Hence, we examined the predictability of BSUA in estimating patient prognosis after chemotherapy in aged individuals with stage IIIB and IV NSCLC.

\section{Method}

\section{Study design and patient recruitment}

This is a single-center retrospective examination, conducted at the West China Hospital, Sichuan University in Chengdu, Sichuan Province, China, between the duration of January 2010 and December 2017. The mediam follow-up period was 28 months. Study eligibility included male stage III B or IV NSCLC patients, $>60$ years of age, who already received the initial course of chemotherapy for NSCLC. In particular, these patients had either ACLC or SCCLC, confirmed with histopathology. In addition, patients with significant renal insufficiency, gout, hyperuricemia, regular systematic use of glucocorticoids, or cyclosporine were eliminated from the study. Our work received ethical approval from the West China Hospital, Sichuan University (No.2018-94).

\section{Measures}

Data regarding the short-term chemotherapy-driven $\mathrm{AE}$ like bone marrow suppression, digestive system reactions, infection, and liver dysfunction were obtained from patient medical records. In addition, survival or ACM data, as of March 1,2019, was collected via phone conversations. Overall survival (OS) represented duration from therapy initiation till death or the last day the patient was documented as alive.Patient demographics like age, smoking habit, diagnosis of chronic diseases, along with tumor 
characteristics like histopathology, clinical stage, radiation therapy, LC surgery, and blood test results prior to chemotherapy were documented and used as essential covariates.

\section{Primary Outcome}

Before chemotherapy, blood was collected from the veins of all participants after fasting for $>8 \mathrm{~h}$. BSUA was quantified in the West China Hospital lab, following routine protocols. Subsequently, all patients were assigned into one of three cohorts, namely, low, moderate, or high, based on the UA quantile (Q1 and Q3) threshold. Low: UA< Q1; moderate: UA:Q1-Q3; and high: UA>Q3.

\section{Statistical analysis}

All data analyses was done with SPSS 26.0 (IBM Corp. Somers, NY, USA). Data are presented as mean \pm standard deviation (SD) or median and interquartile range (IQR), based on data distribution. Categorical variables are expressed as numbers (percentages). Patient baseline characteristics were analyzed with rank-sum, student's t, and Pearson's chi-square tests. Binary Logistic regression analysis examined the link between BSUA and short-term AE after chemotherapy. Cox regression analysis explored the link between BSUA and mortality. Lastly, two-sided $p<0.05$ was the significance threshold.

\section{Results}

\section{Study participant characteristics}

Overall, 317 male NSCLC patients, aged $>60$ years, were enrolled in this study. Among them, the proportion of NSCLC staged IIIB and IV were $18.3 \%$ and $81.7 \%$, respectively. Moreover, confirmed ACLC and SCCLC diagnosis was done in $81.39 \%$ and $18.61 \%$ of patients, respectively. We grouped patients according to the BSUA levels, based on the quartile method; in particular, low: <Q1 (256umol/I); moderate: Q1-Q3 (256umol/l-349.5umol/l); high: >Q3 (349.5umol/l). Furthermore, we demonstrated marked differences in clinical stage and chemotherapeutic regimen among the three cohorts. However, no discernible differences were observed in the patient age, smoking habit, health insurance, chronic diseases, BMI, tissue type, radiation therapy, history of LC surgery, systolic blood pressure, as well as heart and respiration rates (Table 1). 
Table 1

Patient baseline characteristics

\section{Characteristics}

(2)

Age (years),m(iqr)

Smoking status, $n(\%)$

non-smoker

former smoker

current smoker

Health Insurance, n (\%)

no

yes

Number of chronic diseases*, $\mathrm{n}(\%)$

$<3$

$\geq 3$

$\mathrm{BMl}$, mean $\pm \mathrm{SD}$

Tissue type,n(\%)

adenocarcinoma

squamous cell carcinoma

Clinical stage,n(\%)

IIIB

IV

Regimen of chemotherapy,n(\%)

single

combination

Radiation therapy

\begin{tabular}{lll}
$64(80)$ & $128(81.01)$ & $66(83.54)$ \\
\hline $16(20)$ & $30(18.99)$ & $13(16.46)$
\end{tabular}

$\begin{array}{lll}70(87.50) & 137(86.71) & 66(83.54) \\ 10(12.50) & 21(13.29) & 13(16.46)\end{array}$

$22.34 \pm 2.89$

$22.26 \pm 2.57$

$22.80 \pm 4.32$

0.454

0.836

0.03

7(8.75)

32(20.25)

19(24.05)

73(91.25)

126(79.75)

60(75.95)

0.736

0.093

$\begin{array}{lll}46(57.50) & 69(43.67) & 34(43.04) \\ 34(42.50) & 89(56.33) & 45(56.96)\end{array}$

$\mathrm{N}=80 \quad \mathrm{~N}=158 \quad \mathrm{~N}=79$




\begin{tabular}{|c|c|c|c|c|}
\hline Characteristics & $\begin{array}{l}\text { Low BSUA } \\
\mathrm{N}=80\end{array}$ & $\begin{array}{l}\text { Moderate BSUA } \\
N=158\end{array}$ & $\begin{array}{l}\text { High BSUA } \\
N=79\end{array}$ & $P$ \\
\hline no & $59(73.75)$ & $131(82.91)$ & $62(78.48)$ & \\
\hline yes & $21(26.25)$ & $27(17.09)$ & $17(21.52)$ & \\
\hline Patients with lung cancer surgery & & & & 0.163 \\
\hline no & 75(93.75) & $143(90.51)$ & $67(84.81)$ & \\
\hline yes & $5(6.25)$ & 15(9.49) & 12(15.19) & \\
\hline Systolic blood pressure(mmhg),m[iqr & 121.5 & 122 & 125 & 0.917 \\
\hline Heart rate,m(iqr) & 80 & 80.5 & 82 & 0.663 \\
\hline Breathing,m(iqr) & 20 & 20 & 20 & 0.548 \\
\hline \multicolumn{5}{|c|}{$\begin{array}{l}\text { Note: Inter-group statistical analysis was done with Kruskal-Wallis, Pearson's chi-square, Fisher's } \\
\text { exact, or one-way analysis of variance, as appropriate. *chronic illness like hypertension, coronary } \\
\text { heart disease, chronic obstructive pulmonary disease, asthma, digestive system diseases, diabetes } \\
\text { mellitus, thyroid diseases, nervous system diseases, immunological diseases, osteoarticular diseases } \\
\text { and so on (lung cancer, gout, and hyperuricemia excluded). Low BSUA: <256umol/l; Moderate BSUA: } \\
256 \text { umol//-349.5umol/l/; High BSUA: >349.5umol/l. }\end{array}$} \\
\hline
\end{tabular}

\section{Short-term Adverse Effects}

Following initial chemotherapeutic course, the rate of short-term AE like bone marrow suppression, all infection, liver function impairment, digestive reactions were $13.25 \%, 7.26 \%, 5.36 \%$, and $4.1 \%$, respectively. The cohort with moderate BSUA exhibited the lowest prevalence of short-term AE and ACM (Table 2). Moreover, using model 1, we demonstrated that the moderate BSUA is correlated with reduced bone marrow suppression risk $(\mathrm{OR}=0.42,95 \% \mathrm{Cl}: 0.196-0.9 ; \mathrm{p}=0.026)$. Upon adjustment for confounding factors, the binary logistic regression analysis showed that the moderate BSUA was independently linked to a lower bone marrow suppression risk ( $\mathrm{OR}=0.407,95 \% \mathrm{Cl}: 0.178-0.931 ; \mathrm{p}=0.033$, Table 3 ). 
Table 2

Comparison of short-term chemotherapy-driven unfavourable reactions and patient mortality, based on BSUA

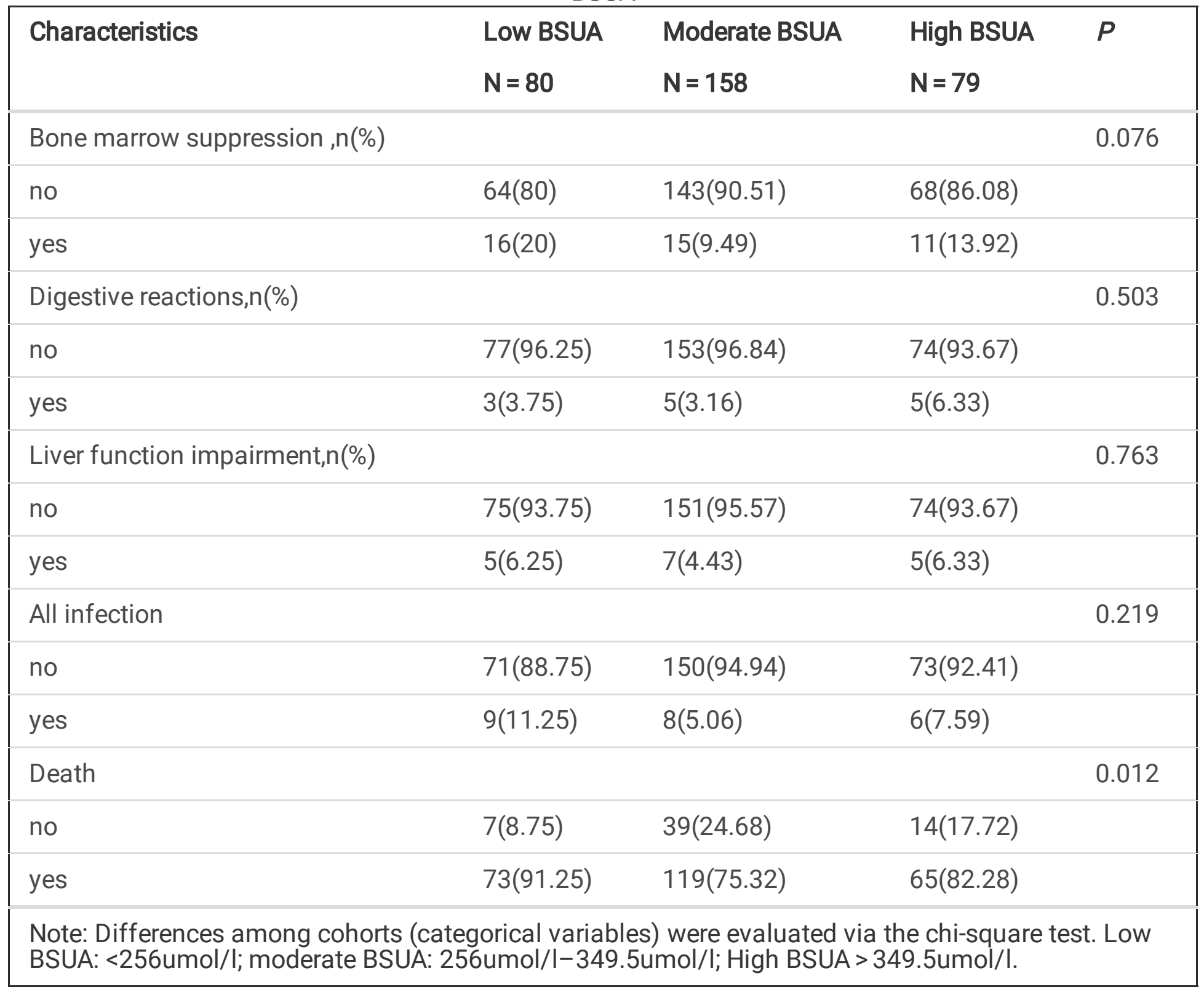


Table 3

The link between BSUA and short-term chemotherapy-related unfavourable reaction risk

\begin{tabular}{lllllll} 
Variable & \multicolumn{2}{l}{ Low BSUA } & \multicolumn{2}{l}{ Moderate BSUA } & \multicolumn{2}{l}{ High BSUA } \\
\cline { 2 - 5 } & P & OR $(95 \%$ & P & OR $(95 \% \mathrm{Cl})$ & P & OR (95\% Cl)
\end{tabular}

\section{Bone marrow}

suppression

$\begin{array}{lllllll}\text { Model } 1 & - & 1 & 0.026 & 0.42(0.196-0.9) & 0.31 & 0.647(0.279-1.499) \\ \text { Model } 2 & - & 1 & 0.033 & 0.407(0.178- & 0.424 & 0.685(0.271-1.732) \\ & & & & 0.931) & & \end{array}$

\section{Digestive \\ reactions}

$\begin{array}{lllllll}\text { Model 1 } & - & 1 & 0.813 & \begin{array}{l}0.839(0.195- \\ 3.602)\end{array} & 0.462 & 1.734(0.4-7.516) \\ & & & & & \\ \text { Model 2* } & - & 1 & 0.993 & \begin{array}{l}1.009(0.161- \\ 6.307)\end{array} & 0.287 & 2.792(0.422- \\ & & & & & & 18.482)\end{array}$

\section{Liver function}

\begin{tabular}{lllllll} 
Model 1 & - & 1 & 0.546 & $\begin{array}{l}0.695(0.214- \\
2.264)\end{array}$ & 0.984 & $1.014(0.282-3.647)$ \\
\hline Model2** & - & 1 & 0.433 & $\begin{array}{l}0.608(0.175- \\
2.107)\end{array}$ & 0.994 & $0.995(0.253-3.916)$
\end{tabular}

\section{All infections}

$\begin{array}{lllllll}\text { Model } 1 & - & 1 & 0.088 & \begin{array}{l}0.421(0.156- \\ 1.136)\end{array} & 0.433 & 0.648(0.219-1.916) \\ \text { Model } 2 & - & 1 & 0.371 & 0.61(0.207-1.8) & 0.953 & 1.037(0.315-3.413)\end{array}$

\section{Note:}

Model 1: non-adjusted.

Model 2: after adjusting for age, smoking status, health insurance, chronic diseases, BMI, tissue type, clinical stage, chemotherapy regimen, radiation therapy, history of LC surgery, systolic blood pressure, as well as heart and respiration rates.

* When the digestive reactions were taken as the outcome index, the sample size of chemotherapy regimens was too small to be included in the analysis.** When the liver function was taken as the outcome index, the sample size of patients with LC surgery was too small to be included in the analysis.

\section{Note}

Differences among cohorts (categorical variables) were evaluated via the chi-square test. Low BSUA: <256umol/l; moderate BSUA: 256umol/I-349.5umol/l; High BSUA > 349.5umol/I. 


\section{Mortality}

As of March 1, 2019, 257 (81.07\%) patients passed away. Based on model 1, moderate BSUA is linked to a reduced risk of $\mathrm{ACM}(\mathrm{HR}=0.673,95 \% \mathrm{Cl}: 0.502-0.901 ; \mathrm{p}=0.008)$. After confounding factors were adjusted, namely age, smoking status, health insurance, chronic diseases, BMI, tissue type, clinical stage, chemotherapy regimen, radiation therapy, history of LC surgery, systolic blood pressure, as well as heart and respiration rates, the Cox regression analysis revealed that the moderate BSUA was strongly correlated with a low ACM risk ( $\mathrm{HR}=0.705,95 \% \mathrm{Cl}: 0.518-0.959 ; \mathrm{p}=0.026$, Table 4).

Table 4

The link between BSUA and all-cause mortality risk

\begin{tabular}{|lllll|}
\hline variable & \multicolumn{2}{l}{ Model 1 } & \multicolumn{3}{c|}{ Model 2 } \\
\cline { 2 - 5 } & P-value & HR (95\% Cl) & P-value & HR (95\% CI) \\
\hline Low BSUA & - & 1 & - & 1 \\
\hline Moderate BSUA & 0.008 & $0.673(0.502-0.901)$ & 0.026 & $0.705(0.518-0.959)$ \\
\hline High BSUA & 0.113 & $0.761(0.544-1.066)$ & 0.230 & $0.805(0.565-1.147)$ \\
\hline Note & & & & \\
$\begin{array}{l}\text { Model 1: unadjusted. } \\
\text { Model 2: after adjusting for age, smoking status, health insurance, chronic diseases, BMl, tissue type, } \\
\text { clinical stage, chemotherapy regimen, radiation therapy, history of LC surgery, systolic blood pressure, } \\
\text { as well as heart and respiration rates. }\end{array}$ \\
\hline
\end{tabular}

\section{Discussion}

Herein, we demonstrated that moderate BSUA is intimately related to a reduced bone marrow suppression and mortality risk. Hence, BSUA has great potential in estimating chemotherapy-driven AE in male NSCLC patients. Moreover, BSUA assessment is highly feasible and requires only a venous blood sample that can be collected during routine blood work at the time of inpatient admission.

Although the physiological effects of uric acid is complex (15-16), excessively high or low BSUA levels can induce damage to normal physiological functions. For instance, elevated BSUA was reported to be strongly correlated with malignancy. Additionally, enhanced nucleic acid turnover, which produces more BSUA, was observed in cases of aggressive cancers, with close association with increased mortality (17). Alternately, diminished BSUA levels may result from apetite loss and cancer-related cachexia, in which case both the oral ingestion and endogenous production of BSUA may be insufficient (9). It is well known that cachexia often suggests a worse prognosis (15). Based on our research results, we propose that moderate BSUA may exert a protective effect on the body, possibly since it indicates that the patient has no significant cachexia, and there is no side effect of high BSUA. However, this is only our inference, and it requires further investigation and validation. 
However, few reports suggest that moderate BSUA is related to chemotherapy-related AE. Hence, further mechanistic studies are warranted to elucidate the true mechanism of moderate BSUA. In our study, patients with low BSUA were employed as the reference cohort. In addition, our observations showed no association between high BSUA and ACM after chemotherapy, which is inconsistent with prior studies (9). This inconsistency may have arisen from the varied threshold selection depending on the multiple BSUA grouping. Additionally, the population selection was also inconsistent.

This is a novel investigation of the correlation between BSUA and chemotherapy-related AE and ACM in hospitalized male NSCLC patients in China. During our study, we encountered certain limitations. First, the baseline functional status of eligible patients modulated their mortality and AE. Unfortunately, this particular data was not available to us and, therefore, could not be included in the analysis. Second,this study did not classify the severity of AE and it only considered AE that caused certain deviations. Third, our research design was retrospective in nature, and, therefore, could have introduced a possible selection bias. Additionally, our sample population was small. Hence, our conclusions would require further investigation, involving a larger sample population, and validation.

\section{Conclusion}

BSUA is intimately linked to chemotherapy-related AE and ACM in male patients with stage IIIB or IV NSCLC.

\section{Abbreviations}

BUSA:baselineserum uric acid; AE; adverse effects ; ACM:all-cause mortality; NSCLC:non-small cell lung cancer;ACLC:adenocarcinoma lung cancer; SCCLC: squamous cell carcinoma lung cancer;LC:lung cancer; OS:overall survival;

\section{Declarations}

\section{Acknowledgements}

We thank all personnel for their contribution in the study.

\section{Author contributions}

$\mathrm{XC}, \mathrm{LH}$ and QH wrote the main manuscript text. YS, JL and BD prepared Table 1-3. All authors reviewed the manuscript.

\section{Funding}

1. Collaborative Innovation Centre of Sichuan for Elderly Care and Health of China (No. YLZBZ1804) and Project of Health and family planning commission of Sichuan Province (CGY2017-101).

2. 2020 Zigong City Key Technology Support Plan (Project No. 2020YLSF19). 
Availability of date and materials

This data is still being analyzed in other directions, and the data can be obtained from the corresponding author.

\section{Ethical approval and consent to participate}

This study was conducted in line with the Declaration of Helsinki and was approved by the Ethical Review Committee of West China Hospital of Sichuan University with the committee's reference number 2017(445) and the registration number is 2018-94. The Ethical Review Committee of West China Hospital of Sichuan Universitywaived the informed consent of this study. All methods were consistent with relevant guidelines and regulations.

\section{Consent for publication}

Not applicable.

\section{Competing interests}

The authors declare that there are no conflicts of interest.

\section{References}

1. Zheng RS, Sun KX, Zhang SW,et al.Report of cancer epidemiology in China ,2015[J].Chin J Oncol, 2019,41(1):19-28.doi:3760/cma.j.issn.0253-3766.2019.01.005.

2. Sung H, Ferlay J,Siegel RL,et al.Global cancer statistics 2020:GLOBOCAN estimates of incidence and mortality worldwide for 36 cancers in 185 countries [J].CA Cancer J Clin, 2021. doi:10.3322/caac.21660.

3. Wang $Z$, Wang $Y$, Zhang $X$,et al. Pretreatment prognostic nutritional index as a prognostic factor in lung cancer: Review and meta-analysis[J]. Clin Chim Acta. 2018,486:303-310. doi: 1016/j.cca.2018.08.030.

4. Gao Y, Zhang H, Li Y, et al. Preoperative increased systemic immune-inflammation index predicts poor prognosis in patients with operable non-small cell lung cancer[J]. Clin Chim Acta. 2018,484:272277.doi:1016/j.cca.2018.05.059.

5. Miller K D , Siegel R L , Lin C C , et al. Cancer treatment and survivorship statistics, 2016[J]. CA: A Cancer Journal for Clinicians, 2016,66:271-289.doi:3322/caac.21349.

6. Siegel, K. Miller, Jemal A. Cancer statistics, 2016[J].CA Cancer J. Clin,2016, 66(1) :7-30.doi: $10.3322 /$ caac. 21332 .

7. Chen S, Yan H, Du J, et al. Prognostic significance of pre-resection albumin/fibrinogen ratio in patients with non-small cell lung cancer: A propensity score matching analysis[J]. Clin Chim Acta. 2018,482:203-208.doi:1016/j.cca.2018.04.012. 
8. Zhu CM, Lian XY, Bi YH,er al. Prognostic value of ribonucleotide reductase subunit M1 (RRM1) in non-small cell lung cancer: A meta-analysis[J]. Clin ChimActa.2018,485:67-

73.doi:1016/j.cca.2018.05.042.

9. Tanriverdi $O$, Cokmert S, Oktay E, et al. Prognostic significance of the baseline serum uric acid level in non-small cell lung cancer patients treated with first-line chemotherapy: a study of the Turkish Descriptive Oncological Researches Group[J]. Medical Oncology, 2014, 31(10):1-7.doi: 1007/s12032-014-0217-z.

10. Willet WC, MacMahon B.Diet and cancer-an overview (second of two parts)[J]. New England Journal of Medicine, 1984, 310(11):697-703.doi:1056/NEJM198403153101106.

11. Wang $\mathrm{H}$, Shan $\mathrm{D}$, Dong $\mathrm{Y}$, et al. Correlation analysis of serum cystatin $\mathrm{C}$, uric acid and lactate dehydrogenase levels before chemotherapy on the prognosis of small-cell lung cancer[J]. Oncol Lett. 2021 Jan;21(1):73.doi:3892/ol.2020.12334.

12. Masi $S$, Georgiopouls $G$, Alexopulos $G$, et al. The conplex relationship between serum uric acid,endothelial function and small vessel remodeling in humans[J].J Clin Med,2020,9(7);2027.doi: 3390/jcm9072027.

13. Ghaemi-Oskouie F, Shi Y . The Role of Uric Acid as an Endogenous Danger Signal in Immunity and Inflammation[J]. Current Rheumatology Reports, 2011, 13(2):160-166.doi: 1007/s11926-011-0162-1.

14. Linder N, R Bützow, Lassus $H$, et al. Decreased xanthine oxidoreductase (XOR) is associated with a worse prognosis in patients with serous ovarian carcinoma[J]. Gynecologic Oncology, 2012, 124(2):311-318. doi:1016/j.ygyno.2011.10.026.

15. Fini MA, Elias A, Johnson RJ, et al. Contribution of uric acid to cancer risk, recurrence, and mortality. Clin Transl Med.2012;1:16-doi:10.1186/2001-1326-1-16.

16. Ames BN, Catheart R, Schwiers $E$, et al. Uric acid provides an antioxidant defense in humans against oxidant- and radical-caused aging and cancer: a hypothesis[J]. Proc Natl Acad Sci USA, 1981;78:6858-doi:10.1073/pnas.78.11.6858.

17. Sevanian A, Davies K J , Hochstein P. Serum urate as an antioxidant for ascorbic acid.[J]. American Journal of Clinical Nutrition, 1991, 54(6):1129-doi:10.1093/ajcn/54.6.1129s. 